\title{
A SIMPLICITY THEOREM FOR AMOEBAS OVER RANDOM REALS
}

\author{
FRED G. ABRAMSON ${ }^{1}$
}

\begin{abstract}
Let $M$ be a countable standard transitive model of ZFC, $\mathcal{Q}$ be an amoeba over $M$, and $r$ be a random real over $M$.

THEOREM. (a) There is no infinite set of reals $X$ contained in the complement of $Q$ with $X \in M[r] ;$ (b) If $\left\{x_{n} \mid n<\omega\right\} \in M[r]$ is a sequence of distinct reals, then for all large enough $n,\left\{x_{i} \mid 2^{n}<i<2^{n+1}\right\} \cap \mathbb{Q} \neq \varnothing$.
\end{abstract}

Introduction. Solovay's by now classical construction ${ }^{2}$ of a model of set theory in which there is no non-Lebesgue-measurable set of reals has prompted a number of unsuccessful attempts to obtain a similar model without recourse to any large cardinal hypothesis. There is a certain plausible approach motivated by the observation that iterated amoeba forcing, à la Martin's axiom, produces a model in which, for any real $p$, almost every real is random over $L(p){ }^{3}$ The stumbling block here has been the failure to obtain a "factoring" lemma, i.e., to analyze the structure of what happens to the forcing algebras involved when they are reduced over a given random real. The basic problem would be to factor an amoeba over a random real.

Part (a) of the Theorem stated in the Abstract says that for $r$ random over $L, \mathbb{Q}$ an amoeba over $L$, and $r \in L[\mathcal{Q}]$, the information about $\mathbb{Q}$ encoded in $r$, or, more specifically, the information about $\mathcal{Q}$ 's complement, is severely limited. $\mathcal{Q}$, of course, contains every real in $L$. Part (b) gives a certain sense in which $\mathscr{Q}$ contains a thick portion of the reals in $L[r]$ also. (The function $n \mapsto 2^{n}$ is not a tight bound. The point is that there is a certain thickness obtained independent of the choice of $\mathcal{Q}, r$, and the sequence of reals.)

Aside from usual forcing technology and absoluteness considerations, the main instrument of the proof is a combinatorial, measure-theoretic lemma about "covering" sequences of measurable functions with sets of small measure. Before getting into the proofs themselves, let us make the notions involved precise.

Terminology and notation. By the "real numbers" we mean Cantor space, i.e., $2^{\omega}$ endowed with the usual topology. It will sometimes be useful to make $2^{\omega}$ correspond with the usual middle-third Cantor set. To this end, define $\nu(x)=\Sigma_{x(i)=1} 2$. $3^{-(i+1)}$ for $x \in 2^{\omega}$. By "Lebesgue measure" on the reals we mean the usual

Received by the editors July 14, 1978.

AMS (MOS) subject classifications (1970). Primary 02K05, 28A20; Secondary 02K30, 04A15.

Key words and phrases. Amoeba forcing, Solovay forcing, random reals.

${ }^{1}$ This research supported, in part, by NSF Grant Number MCS76-07025.

${ }^{2}$ See Solovay [1970].

${ }^{3}$ For general background on iterated forcing and Martin's axiom, see Martin-Solovay [1970]. 
probability measure $\mu$ such that for each $i<\omega, \mu(\{x \mid x(i)=0\})=\mu(\{x \mid x(i)=1\})$ $=\frac{1}{2}$.

For $h: \omega \rightarrow$ the reals of ordinary real analysis and $A \subseteq \omega$, we say that $A$ is $h$-thick if for all large enough $n,\{i \in \omega \mid h(n) \leqslant i<h(n+1)\} \cap A \neq \varnothing$.

Let $\bar{\epsilon}$ be a fixed rational, $0<\bar{\epsilon}<1 . \mathscr{P}_{\bar{\epsilon}}$ is $\left\{X \subseteq 2^{\omega} \mid X\right.$ open and $\left.\mu(x)<\bar{\epsilon}\right\}$, partially ordered so that $X$ is stronger than $Y$ iff $X \supseteq Y$. $\delta$ is $\left\{X \subseteq 2^{\omega} \mid X\right.$ closed and $\mu(X)>0\}$, partially ordered so that $X$ is stronger than $Y$ iff $X \subseteq Y$. For the remainder of this paper, let $M$ be a fixed countable standard transitive model of ZFC. $\mathcal{Q}$ is an amoeba over $M$ means that $\mathcal{Q}$ is $M$-generic on $\left(\mathscr{P}_{\bar{\epsilon}}\right)^{M}$. $\mathcal{Q}$ is freely thought of either as an open set of reals of measure $\bar{\epsilon}$, or as a code for such a set, and for $x$ any real (not necessarily in $M[\mathbb{Q}]$ ), the meaning of " $x \in \mathbb{Q}$ " is well defined and absolute. Simple density arguments show that $Q$ contains every set of measure 0 coded in $M . r$ is $M$-random means that $r$ is the real determined by an $M$-generic object on $(\delta)^{M}$. $r$ is $M$-random iff $r$ lies in every set of reals of measure 1 coded in $M$.

For the Theorem stated above to apply nontrivially to factoring an amoeba over a random real, it would have to be the case that in $M[Q]$ there is a random real $r$ such that $\left(2^{\omega} \cap M[r]\right)-\mathbb{Q}$ is infinite. Observation 1 below shows that this is always the case.

Notation. For any real $r$ and $n<\omega, r \oplus n$ is the real given by $(r \oplus n)(i)=r(i)$ iff $i \neq n$.

Observation 1. Let $\mathbb{Q}$ be an amoeba over $M$. Then in $M[\mathbb{Q}]$ there is a real $r$ such that $\{n \mid r \oplus n \notin Q\}$ is infinite, and any such real is $M$-random.

Proof. Work in $M[\mathbb{Q}]$. Let $X=\{r \mid\{n \mid r \oplus n \notin \mathbb{Q}\}$ is infinite $\}$. We show, in fact, that $\mu(X)>0$. Let $Y_{m}=\{x \mid \forall n>m(r \oplus n \in \mathbb{Q})\} .2^{\omega}-X=\cup_{m<\omega} Y_{m}$. If $\mu(X)=0$ then let $m$ be such that $\mu\left(Y_{m}\right)>\bar{\epsilon}$. Now, for all $x \in Y_{m}, x \oplus(m+1) \in$ $\mathbb{Q}$, and it is routine to see that $\mu\left(\left\{x \oplus(m+1) \mid x \in Y_{m}\right\}\right)=\mu\left(Y_{m}\right)$, so $\mu(\mathbb{Q})>\bar{\epsilon}$, a contradiction.

Let $r \in X$, and take $n$ such that $r \oplus n \notin \mathbb{Q}$. Then $r \oplus n$ is $M$-random as it can be in no set of measure 0 coded in $M$, each of these being contained in $\mathcal{Q}$. It is easy to check that as $r \oplus n$ is $M$-random, so must be $r$.

Observation 1 gives us an example of an infinite sequence of reals in $M[r]$, namely $\left\{x_{n}=r \oplus n \mid n<\omega\right\}$, such that $\left\{x_{n}\right\}-\mathbb{Q}$ is infinite but, by the Theorem we shall prove, it has no infinite subset in $M[r]$.

The proof of the Theorem proceeds by giving an appropriate representation of reals in $M[r]$ as Borel functions in $M$ and then by proving a "covering" principle for sequences of measurable functions. As there will be several different models of set theory involved, it will be necessary to speak of "codes" for Borel functions. Rather than going into details, suffice it to say that we fix a way of representing (total) Borel functions: $2^{\omega} \rightarrow 2^{\omega}$ by elements of $2^{\omega}$ in such a way that the predicate $C(x) \equiv$ " $x$ is a code for a total Borel function" is $\Pi_{1}^{1}$ and the predicate $F(x, y, z)$ $\equiv$ "the function coded by $x$ applied to argument $y$ yields value $z$ " is $\Delta_{1}^{1}$ in the sense that there are $\Sigma_{1}^{1}$ and $\Pi_{1}^{1}$ predicates $P$ and $Q$ such that whenever $C(x)$ holds then for all $y$ and $z, F(x, y, z) \leftrightarrow P(x, y, z) \leftrightarrow Q(x, y, z)$. Thus $C$ and $F$ are absolute 
with respect to standard transitive models of $\mathrm{ZF}$.

Notation. For $\mathcal{P}$ a (description of) a partial ordering, we write $p \mathbb{t}_{\mathscr{\rho}} \mathscr{F}$ to mean that $M \vDash p \in \mathscr{P}$ and $p$ weakly forces $\mathscr{F}$ over $\mathscr{P}$.

Proposition 2. Suppose $X \Vdash_{\delta} t \in 2^{\omega}$. Then there is $x \in M$ such that $C(x)$ and $X$ $\mathbb{}_{\delta} F(x, \mathbf{r}, t)$, where $\mathbf{r}$ is the canonical name for the real given by the generic object on $\mathcal{S}$.

Proof. Work in $M$. For each $i<\omega$, let $\left\{X_{i j} \mid j<\omega\right\}$ be a maximal antichain of $\left\{Y \subseteq X \mid Y \mathbb{}_{\delta} t(i)=0\right.$ or $\left.Y \mathbb{}_{\delta} t(i)=1\right\}$. Let $W=\left\{(i, j) \mid X_{i j}\right.$ $\left.\mathbb{}_{\delta} t(i)=1\right\}$. Take $x$ to be a code for the function $y \mapsto\{i \mid \exists j[(i, j) \in W \wedge y \in$ $\left.X_{i j}\right]$ ). Clearly, for $r M$-random and $r \in X, F\left(x, r, E_{r}(t)\right)$ holds, where $E_{r}(t)$ is the evaluation of the term $t$ in $M[r]$.

Definition. Let $\left\{f_{n} \mid n<\omega\right\}$ be a sequence of functions: $2^{\omega} \rightarrow 2^{\omega}$, and let $X, Y \subseteq$ $2^{\omega}$. $Y$ covers $X$ with respect to $\left\{f_{n}\right\}$ iff $\mu\left(\left\{x \in X \mid \exists n<\omega\left[f_{n}(x) \in Y\right]\right\}\right)=\mu(X)$. X is easily covered with respect to $\left\{f_{n}\right\}$ iff $\forall \epsilon>0 \exists Y[\mu(Y)<\epsilon \wedge Y$ covers $X$ with respect to $\left\{f_{n}\right\}$ ].

The following is purely a theorem of real analysis in ZFC.

LemMa 3. Let $\left\{f_{n} \mid n<\omega\right\}$ be a sequence of measurable functions: $X \rightarrow 2^{\omega}, X \subseteq 2^{\omega}$, such that $\forall x \in X \forall i, j \in \omega\left(i \neq j \rightarrow f_{i}(x) \neq f_{j}(x)\right)$. Then (a) $X$ is easily covered with respect to $\left\{f_{n}\right\}$. Moreover, (b) if $\epsilon>0$ there is $X^{\prime} \subseteq X$ such that $\mu\left(X^{\prime}\right)=\mu(X)$ and an open set $U$ with $\mu(U)<\epsilon$ such that for all $x \in X^{\prime},\left\{i \in \omega \mid f_{i}(x) \in U\right\}$ is $\left(n \mapsto n^{5} / \epsilon\right)$-thick.

REMARK. (a) is not particularly obvious, even for very simple sequences $\left\{f_{n}\right\}$. The reader may wish to consider some examples like $f_{n}(x)=(1-1 /(n+2)) \cdot x$ for $0<x<1$. (In this example we are taking "reals" to mean the usual unit interval rather than $2^{\omega}$. It is not hard to see that Lemma 3 applies also in this case.)

Proof of Lemma 3 . We prove (b) which easily yields (a). It is safe to assume $\epsilon<1$, or else just take $X^{\prime}=X$ and $U=2^{\omega}$. Also, we can assume $\mu(X)>0$, otherwise take $X^{\prime}=U=\varnothing$. For each $n<\omega$ we construct $X_{n} \subseteq X$ and an open set $U_{n}$ such that:

(i) $\mu\left(X_{n}\right) \geqslant \mu(X) \cdot\left(1-1 /(n+2)^{2}\right)$,

(ii) $\mu\left(U_{n}\right)<\epsilon \cdot 1 /(n+2)^{2}$,

(iii) $\forall x \in X_{n} \exists i\left[(n+2)^{5} / \epsilon \leqslant i<(n+3)^{5} / \epsilon \wedge f_{i}(x) \in U_{n}\right]$.

Given such $X_{n}$ and $U_{n}$, as $\sum_{n=0}^{\infty}(n+2)^{-2}<\infty$, taking $X^{\prime}=\left\{x \in X \mid x \in X_{n}\right.$ for all large enough $n\}, \mu\left(X^{\prime}\right)=\mu(X)$. Also, as $\sum_{n=0}^{\infty}(n+2)^{-2}<1$, taking $U=$ $\cup_{n<\omega} U_{n}, \mu(U)<\epsilon$. Finally, for $x \in X^{\prime}$, if $m$ is such that $x \in X_{n}$ whenever $n>m$, we have that whenever $n>m+2$,

$$
\left\{i \mid n^{5} / \epsilon \leqslant i<(n+1)^{5} / \epsilon\right\} \cap\left\{i \mid f_{i}(x) \in U\right\} \neq \varnothing .
$$

Fix $n$. Let $I=\left\{i \mid(n+2)^{5} / \epsilon \leqslant i<(n+3)^{5} / \epsilon\right\}$. For $i \in I$, let $\hat{f}_{i}=\nu \cdot f_{i} \cdot \hat{f}_{i}$ is a measurable real-valued (in the ordinary sense of real analysis) function on $X$. Let $A \subseteq X$ be compact with $\mu(A) \geqslant \mu(X) \cdot\left(1-\frac{1}{4} \cdot(n+2)^{-2}\right)$. By Lusin's theorem, there is a compact $B \subseteq A$ with $\mu(B) \geqslant \mu(X) \cdot\left(1-\frac{1}{2} \cdot(n+2)^{-2}\right)$ such that for 
each $i \in I, \hat{f}_{i}$ is continuous on $B$. Thus the function $x \mapsto \inf \left\{\left|\hat{f}_{i}(x)-\hat{f}_{j}(x)\right| \mid i, j \in I\right.$, $i \neq j\}$ is a continuous, strictly positive function on $B$. So $\delta \equiv \inf \left\{\left|\hat{f}_{i}(x)-\hat{f}_{j}(x)\right| \mid i, j\right.$ $\in I, i \neq j, x \in B\}>0$. Let $Y_{0}, \ldots, Y_{k-1}$ be a pairwise-disjoint collection of basic open neighborhoods of $2^{\omega}$ such that each has measure $1 / k, \cup_{j<k} Y_{j}=2^{\omega}$, and the diameter of $\nu\left[Y_{j}\right]<\delta$. So, for any $x \in B$ and $j<k$ there is at most one $i \in I$ with $f_{i}(x) \in Y_{j}$.

Claim. Suppose $W \subseteq B$. Then there is $j<k$ such that

$$
\mu\left(\left\{x \in W \mid \exists i \in I\left[f_{i}(x) \in Y_{j}\right]\right\}\right) \geqslant \overline{\bar{I}} \cdot \mu(W) / k .
$$

ReMARK. To understand the point of this claim, imagine that $\mu(W)=1$. Then it says that the amount of $W$ that $Y_{j}$ "covers" is very large compared to the size of $Y_{j}$, namely, $\bar{I}$ times as large. This is the essential combinatorial idea behind Lemma 3.

Proof of Claim. Let $Z_{i j}=\left\{x \in W \mid f_{i}(x) \in Y_{j}\right\}$. So for each $j$, if $i \neq i^{\prime}$ then $Z_{i j} \cap Z_{i^{\prime} j}=\varnothing$. Thus

$$
\begin{gathered}
\mu\left(\left\{x \in W \mid \exists i \in I\left[f_{i}(x) \in Y_{j}\right]\right\}\right)=\sum_{i \in I} \mu\left(Z_{i j}\right) . \\
\sum_{j<k} \sum_{i \in I} \mu\left(Z_{i j}\right)=\sum_{i \in I} \sum_{j<k} \mu\left(Z_{i j}\right)=\overline{\bar{I}} \cdot \mu(W),
\end{gathered}
$$

as $\left\{Z_{i j} \mid j<k\right\}$ is a partition of $W$. So for some $j<k$ it must be that $\sum_{i \in I} \mu\left(Z_{i j}\right)>$ $\bar{I} \cdot \mu(W) / k . \quad \square$ (for claim)

Now, construct a (finite) sequence of subsets of $k, K_{0} \subseteq K_{1} \subseteq \cdots \subseteq K_{s}$ as follows. Let $K_{0}=\varnothing$. Assume $K_{t}$ has been defined. Let $V_{t}=\left\{x \in B \mid \exists i \in I\left[f_{i}(x)\right.\right.$ $\left.\left.\in \cup_{j \in K_{t}} Y_{j}\right]\right\}$. If $\mu\left(V_{t}\right) \geqslant \mu(X) \cdot\left(1-(n+2)^{-2}\right)$, then let $s=t$ and $K_{t}$ is the last element of our sequence. Otherwise, let $W=B-V_{t}$ and let $K_{t+1}=K_{t} \cup\{j\}$ where $j$ is chosen so that $\mu\left(\left\{x \in W \mid \exists i \in I\left[f_{i}(x) \in Y_{j}\right]\right\}\right) \geqslant \overline{\bar{I}} \cdot \mu(W) / k$. Obviously $j \notin K_{t}$. As $\mu(W) \geqslant \mu(X) \cdot\left(1 / 2(n+2)^{2}\right)$,

$$
\mu\left(V_{t+1}\right) \geqslant \mu\left(V_{t}\right)+(\overline{\bar{I}} / k) \cdot \mu(X) \cdot\left(1 / 2(n+2)^{2}\right) .
$$

As $k$ is finite, the iterative procedure eventually yields $K_{s}$ such that $\mu\left(V_{s}\right) \geqslant \mu(X)$ $\cdot\left(1-(n+2)^{-2}\right)$.

$$
\mu(X) \geqslant \mu\left(V_{s}\right) \geqslant s \cdot(\overline{\bar{I}} / k) \mu(X) \cdot\left(1 / 2(n+2)^{2}\right),
$$

so letting $U_{n}=\cup_{j \in K}, Y_{j}$, we have $\mu\left(U_{n}\right)=s / k \leqslant 2(n+2)^{2} / \overline{\bar{I}}$. As $\overline{\bar{I}}>2(n+$ $2)^{4} / \epsilon, \mu\left(U_{n}\right)<\epsilon /(n+2)^{2}$. Thus we have (ii). Now, taking $X_{n}=V_{s}$, we immediately get (i) and (iii). $\square$ (for Lemma 3)

We now prove the Theorem stated in the Abstract. (b) clearly yields (a), so let $\left\{x_{n} \mid n<\omega\right\} \in M[r]$ be a sequence of distinct reals. Let $t$ be a term such that $E_{r}(t)$ is this sequence, and let $X_{1}$ be such that $r \in X_{1}$ and $X_{1} \mathbb{\Vdash}_{\delta} t$ is a sequence of distinct reals. Let $t_{n}$ be the term saying "the $n$th element of $t$ ". Now, working 'in $M$, for each $n$, let $x_{n}$ be such that $C\left(x_{n}\right) \wedge X_{1} \mathbb{1}_{\S} F\left(x_{n}, \mathbf{r}, t_{n}\right)$. For $x \in X_{1}$, let $f_{n}(x)=$ the unique $z$ such that $F\left(x_{n}, x, z\right)$. For $m, n \in \omega, m \neq n$, let $A_{m, n}=\left\{x \mid f_{m}(x)=\right.$ $\left.f_{n}(x)\right\}$. Suppose $\mu\left(A_{m, n}\right)>0$. Then $M \vDash \forall x \in A_{m, n} \forall z\left[F\left(x_{m}, x, z\right) \leftrightarrow F\left(x_{n}, x, z\right)\right]$. Thus $A_{m, n} \Vdash_{\S} t_{m}=t_{n}$, for, if $r^{\prime}$ is $M$-random and $r^{\prime} \in A_{m, n}$, by absoluteness for $\Pi_{1}^{1}$ 
sentences, $M\left[r^{\prime}\right] \vDash \forall x \in A_{m, n} \forall z \quad\left[F\left(x_{m}, x, z\right) \leftrightarrow F\left(x_{n}, x, z\right)\right]$ and, as $A_{m, n} \subseteq X_{1}$, $M\left[r^{\prime}\right] \vDash F\left(x_{m}, r^{\prime}, t_{m}\right) \wedge F\left(x_{n}, r^{\prime}, t_{n}\right)$. This contradicts that $X_{1} \mathbb{t}_{\delta} t$ is a sequence of distinct reals. Thus $\mu\left(A_{m, n}\right)=0$. Let $X=X_{1}-\cup\left\{A_{m, n} \mid m, n \in \omega, m \neq n\right\}$. As $\mu(X)=\mu\left(X_{1}\right), r \in X$.

Now, let

$$
\mathscr{D}=\left\{W \in \mathscr{P}_{\bar{\epsilon}} \mid \exists X^{\prime} \subseteq X \exists \epsilon>0\right.
$$

$$
\left.\left[\mu\left(X^{\prime}\right)=\mu(X) \wedge \forall x \in X^{\prime}\left(\left\{i \mid f_{i}(x) \in W\right\} \text { is }\left(n \mapsto n^{5} / \epsilon\right) \text {-thick }\right)\right]\right\} \text {. }
$$

Lemma 3(b) shows that $\mathscr{D}$ is dense in $\mathscr{P}_{\epsilon}$. As $\mathcal{Q}$ is $M$-generic on $\mathscr{P}_{\varepsilon}$, there is $W \in \mathscr{D}$ with $W \subseteq \mathbb{Q}$. Let $X^{\prime}, \epsilon$ be as in the definition of $\mathscr{D}$ for $W$. As $\mu\left(X^{\prime}\right)=$ $\mu(X), r \in X^{\prime}$. The statement $\forall x \in X^{\prime}\left(\left\{i \mid f_{i}(x) \in W\right\}\right.$ is $\left(n \mapsto n^{5} / \epsilon\right)$-thick) is $\Pi_{1}^{1}$, so by absoluteness we get that $M[r] \vDash\left\{i \mid x_{i} \in W\right\}$ is $\left(n \mapsto n^{5} / \epsilon\right)$-thick. Since for large enough $n$ there is always an $m$ with $2^{n}<m^{5} / \epsilon<(m+1)^{5} / \epsilon<2^{n+1}, M[r] \vDash\left\{i \mid x_{i}\right.$ $\in W\}$ is $\left(n \mapsto 2^{n}\right)$-thick. As $W \subseteq \mathbb{Q}$, we are done. $\square$ (for Theorem)

\section{REFERENCES}

D. A. Martin and R. M. Solovay [1970], Internal Cohen extensions, Ann. Math. Logic 2, 143-178.

R. M. Solovay [1970], A model of set theory in which every set of reals is Lebesgue measurable, Ann. of Math. (2) 92, 1-56.

Department of Mathematics, University of Wisconsin-Milwaukee, Milwaukee, Wisconsin 53201

Current address: Department of Mathematics, Stanford University, Stanford, California 94305 